\title{
On the convergence of the Escalator Boxcar Train
}

\author{
Åke Brännström ${ }^{\dagger \ddagger} \quad$ Linus Carlsson ${ }^{\dagger} \quad$ Daniel Simpson ${ }^{\S}$
}

September 27, 2018

\begin{abstract}
The Escalator Boxcar Train (EBT) is a numerical method that is widely used in theoretical biology to investigate the dynamics of physiologically structured population models, i.e., models in which individuals differ by size or other physiological characteristics. The method was developed more than two decades ago, but has so far resisted attempts to give a formal proof of convergence. Using a modern framework of measure-valued solutions, we investigate the EBT method and show that the sequence of approximating solution measures generated by the EBT method converges weakly to the true solution measure under weak conditions on the growth rate, birth rate, and mortality rate. In rigorously establishing the convergence of the EBT method, our results pave the way for wider acceptance of the EBT method beyond theoretical biology and constitutes an important step towards integration with established numerical schemes.
\end{abstract}

Key words. Escalator boxcar train, EBT, convergence, physiologically structured population models, PSPM, measure-valued solutions, transport equation

AMS subject classifications. 65M12, 28A33, 92B05

\section{Introduction}

The population dynamics of ecological and biological systems are often described by an ordinary differential equation of the form

$$
\frac{1}{N} \frac{d N}{d t}=\beta(N)-\mu(N)
$$

where $N=N(t)$ is the total population size at time $t, \beta(N)$ is the birth rate, and $\mu(N)$ is the mortality rate, both of which depends on the population size. The key assumption in this type of model is that every individual in the population is identical. This is clearly unreasonable in many situations, including cases where the gap between birth size and reproductive size is important. A more accurate description of the population dynamics can be given by physiologically structured population models (see e.g., [18]). In these models, the birth rates, death rates, and growth rates of individuals depend on their physiological state $x \in \Omega$, where $\Omega$ is the set of admissible states. In general, these states can represent any aspects of individual physiology such as age, size, mass, height, or girth. For the purpose of this manuscript, we will work with a one-dimensional state space that we think of as representing individual size, but other interpretations are possible and, as we note in the concluding discussion, we expect that our results can easily be extended to higher-dimensional state manifolds.

\footnotetext{
${ }^{\dagger}$ Department of Mathematics and Mathematical Statistics, SE-90187 Umeå, Sweden.

${ }^{\ddagger}$ Evolution and Ecology Program, International Institute for Applied Systems Analysis, A-2361 Laxenburg, Austria.

${ }^{\S}$ Department of Mathematical Sciences, Norwegian University of Science and Technology, N-7491 Trondheim, Norway
} 
In order to specify a physiologically structured population model, we need explicit representations for the mortality, growth, and fecundity rates of individuals as well as the initial population structure. We assume that these rates are respectively on the form $\mu\left(x, E_{t}\right), g\left(x, E_{t}\right)$, and $\beta\left(x, E_{t}\right)$, where $x$ is the size (or more generally the state) of the individual and $E_{t}$ is the environment that individuals experiences at time $t$. The environment is a key factor in the formulation of physiologically structured population models and can, for example, represent the total amount of nutrient available at time $t$ or the size-specific predation rate, see e.g., [18, 5]. While the environment is often low-dimensional, it could potentially be infinite-dimensional as would for example be the case for the shading profile in a forest. Finally, we assume that all new individuals have the same birth size $x_{b}$. With these assumptions, one can show (see e.g., [5]) that the density $u(x, t)$ of individuals of state $x$ at time $t$ is given by the first order, non-linear, non-local hyperbolic partial differential equations with non-local boundary condition

$$
\begin{aligned}
\frac{\partial}{\partial t} u(x, t)+\frac{\partial}{\partial x}\left(g\left(x, E_{t}\right) u(x, t)\right) & =-\mu\left(x, E_{t}\right) u(x, t), \\
g\left(x_{b}, E_{t}\right) u\left(x_{b}, t\right) & =\int_{x_{b}}^{\infty} \beta\left(\xi, E_{t}\right) u(\xi, t) d \xi, \\
u(x, 0) & =u_{0}(x),
\end{aligned}
$$

in which we assume that $x_{b} \leq x<\infty$ and $t \geq 0$.

The first numerical method designed specifically for solving physiologically structured population models was the inventively named Escalator Boxcar Train (EBT) 4]. Rather than approximating the solution directly, it approximates the measure induced by the solution. Regardless of its unconventional solution methodology, the EBT method is widely used by theoretical biologists (see e.g., [2, 13, 19, 20]). One of the reasons for the popularity of the EBT method can be ascribed to the simple biological interpretation of the components of the scheme: the state-space is partitioned into initial cohorts and, for the $i$ th cohort, the EBT method tracks its size $N_{i}(t)$ and the location of its centre of mass $X_{i}(t)$ (see e.g., [5]). The solution measure $d \zeta_{t}:=u(t, x) d x$ is then approximated by

$$
d \zeta_{t} \approx d \zeta_{t}^{N} \equiv \sum_{i=B}^{N} N_{i}(t) \delta_{X_{i}(t)}
$$

where $\delta_{x}$ is the Dirac measure concentrated at $x$. The dynamics of the functions $N_{i}$ and $X_{i}$ will be defined in Sect. 2. The boundary cohort corresponding to $i=B$ is treated differentially from the other cohorts to account for newborn individuals. In the original formulation, 4], this included terms correcting for changes in the average mass arising from the inflow of newborn individuals. For completeness, we consider the original definition of the boundary cohort in Sect. 4.

The convergence of the EBT method has remained an open question since the method was first introduced in 1988. The most successful analysis was performed by de Roos and Metz [6] in 1991. They studied how well the EBT method approximates integrals of the form $\int_{\Omega} \psi(x) u(x, t) d x$ for smooth functions $\psi$, assuming that cohorts are not internalized (see Sect. 22). The result does not assert the convergence of the EBT method but rather, in the language used by de Roos and Metz, that the EBT method consistently approximates integrals of the solution to (11). One reason for the lack of progress is that the usual analytical techniques for analyzing finite element and finite difference schemes are not immediately applicable to the measure-valued case. Over the last two decades, however, the theory of structured population models has been extensively developed [12, 9, 17, 8, 10, 11, 14], and for the first time a full analysis of the EBT method is within our reach.

The aim of this paper is to rigorously prove the convergence of the EBT method. We show that the EBT method converges under far weaker conditions on the growth, death and birth functions than the conditions assumed by de Roos and Metz [6]. Our arguments build on recent theoretical developments by Gwiazda et al. [14 that extend the classical concept of weak solutions to measuredvalued solutions. In the following section, we describe the EBT method in full detail, define weak 
convergence of measures, and define weak solutions to the physiologically structured population model (11). In Sect. 3 we prove the convergence of the EBT method with dynamics of the boundary cohort as introduced in this paper. Our convergence result is then extended to the original definition of the boundary cohort in Sect. 4. We conclude by placing our results into context and by highlighting promising directions for future work. Theorem 14] and Theorem 16] are the main results of this paper.

\section{The Escalator Boxcar Train}

The EBT method is a numerical scheme for solving physiologically structured population models (PSPMs, see e.g., [18]). While there are many possible formulations of PSPMs, several of which are described in the excellent book by Metz and Diekmann [18, we consider the numerical solution of the one-dimensional PSPM with a single birth state $x_{b}$ defined by (1a), (1b), and (1c). The EBT method determines an approximate measured-valued solution $\zeta_{t}^{N}$ to the PSPM as a linear combination of Dirac measures,

$$
\zeta_{t}^{N} \equiv \sum_{i=B}^{N} N_{i}(t) \delta_{X_{i}(t)} .
$$

Each of the terms in the approximation can be interpreted biologically as a cohort composed of $N_{i}$ individuals with average individual state (e.g., size) $X_{i}$ at time $t$. As individuals give rise to offspring with state $x_{b}$ at birth, we need different definitions for internal cohorts and the boundary cohort.

The internal cohorts are numbered $i=B+1, \ldots, N$. These cohorts are chosen at time $t=0$ so that $\zeta_{0}^{N}$ converges weakly to the initial data $u_{0}(x) d x$ as $N \rightarrow \infty$. This is always possible since finite linear combinations of Dirac measures are dense in the weak topology [1, Volume II, p. 214]. Thus, we need not restrict ourselves to initial data prescribed by a function $u_{0}(x)$, but can extend our analysis to general positive Radon measures $\nu_{0}$. Without loss of generality, we will assume that the total mass $\zeta_{0}^{N}\left(\left[x_{b}, \infty\right)\right)=\nu_{0}\left(\left[x_{b}, \infty\right)\right)$ for all $N$. The boundary cohort is the cohort with the lowest index $B$. At time $t=0, B=0$ and we assume that $N_{0}(0)=0$ and $X_{0}(0)=x_{b}$. As time progresses, additional cohorts with negative index will be created through the process of internalization described further below.

The dynamics of the internal cohorts are given by

$$
\begin{aligned}
\frac{d N_{i}}{d t} & =-\mu\left(X_{i}, \zeta^{N}\right) N_{i}, \\
\frac{d X_{i}}{d t} & =g\left(X_{i}, \zeta^{N}\right),
\end{aligned}
$$

where we have assumed a direct dependence of the vital rates on the solution measure, $\zeta^{N}=\zeta_{t}^{N}$, to represent environmental feedback. Similarly, but in contrast to the original formulation of the EBT method by de Roos [4], the dynamics of the boundary cohorts follow

$$
\begin{aligned}
\frac{d N_{B}}{d t} & =-\mu\left(X_{B}, \zeta^{N}\right) N_{B}+\sum_{i=B}^{N} \beta\left(X_{i}, \zeta^{N}\right) N_{i}, \\
\frac{d X_{B}}{d t} & =g\left(X_{B}, \zeta^{N}\right),
\end{aligned}
$$

where the sum is taken over all cohorts including the boundary cohort. This sum reflects the offspring produced by the total population. In line with their biological interpretations, we henceforth assume that all vital rates, the mortality rate $\mu$, the fecundity rate $\beta$, and the growth rate $g$, are non-negative.

With the EBT method defined as above, both the width and the number of individuals in the boundary cohort will increase over time which eventually introduces an unacceptably large approximation error. For this reason, the boundary cohort must be internalized sufficiently often. This implies that the number of cohorts will increase following internalization. The new boundary cohort 
is at the time $t$ of the internalization given by $N_{B}(t)=0$ and $X_{B}(t)=x_{b}$, where $B$ equals the index of the old boundary cohort decremented one step. At the same instant, the previous boundary cohort becomes an internal cohort. To prevent the number of internal cohorts from exceeding computationally acceptable bounds, internal cohorts may be removed when the number of individuals has declined sufficiently. Removal of internal cohorts is important for numerical implementation but will not be considered in this manuscript.

The EBT method differs from traditional numerical schemes in that it aims to approximate the solution as a measure of point masses. Before we can discuss the convergence of the EBT method, it is necessary to extend the classical concept of a weak solution to measures. This extension builds on earlier work by Gwiazda et al. [14] (see also [3, 15]) and Chapter 8 of the monograph [1]. We will work with the cone all finite positive Radon measures denoted $\mathcal{M}_{+}(\Omega)$, where $\Omega$ is a metric space consisting of all admissible individual states. In our presentation, we assume $\Omega=\left[x_{b}, \infty\right]$ and we think of $x \in \Omega$ as the size of an individual. An important reason for working with finite Radon measures is that their behavior at infinity is tightly controlled: for each $\epsilon>0$, there exists a compact set $K_{\epsilon}$ such that $\mu\left(\Omega \backslash K_{\epsilon}\right)<\epsilon$.

Since the EBT method approximates the true solution as a measure of point masses, the natural mode of convergence on $\mathcal{M}_{+}(\Omega)$ is weak convergence 1 :

Definition 1. A sequence of measures $\left\{\mu_{k}\right\}$ on $\Omega$ converges weakly to a measure $\mu$ if

$$
\int_{\Omega} \phi(x) d \mu_{k}(x) \rightarrow \int_{\Omega} \phi(x) d \mu(x),
$$

as $k \rightarrow \infty$ for all bounded continuous real functions $\phi$ on $\Omega$.

The weak convergence defined above induces a topology associated with the Kantorovich-Rubinstein metric:

$$
\rho(\mu, \nu)=\sup \left\{\int_{\Omega} \phi(x) d(\mu-\nu) \mid \phi \in C_{0}^{\infty}(\mathbb{R}),\|\phi\|_{W^{1, \infty}} \leq 1\right\},
$$

in which $\|\phi\|_{W^{1, \infty}}=\|\phi\|_{L^{\infty}}+\left\|\phi^{\prime}\right\|_{L^{\infty}}$. This is also known as the flat metric. With this metric, $\mathcal{M}_{+}(\Omega)$ is a complete metric space (see [14, Def. 2.5]).

Analogously to weak convergence, we define weak continuity as follows:

Definition 2. A mapping $\zeta_{t}: \mathbb{R}_{+} \rightarrow \mathcal{M}_{+}(\Omega)$ is weakly continuous in time if, for all bounded continuous real functions $\phi$ on $\Omega$,

$$
\int_{\Omega} \phi(x) d \zeta_{t}
$$

is continuous in the classical sense as a function of $t$.

With these two topological notions in place, we are in position to define measure-valued solutions to the PSPM (1):

Definition 3. A mapping $\zeta_{t}:[0, T] \rightarrow \mathcal{M}_{+}([0, \infty))$ is a weak solution to (1) up to time $T$ if $\zeta_{t}$ is weakly continuous in time and

$$
\begin{aligned}
& \int_{x_{b}}^{\infty} \phi(x, T) d \zeta_{T}(x)-\int_{x_{b}}^{\infty} \phi(x, 0) d \nu_{0}(x)= \\
& \int_{0}^{T} \int_{x_{b}}^{\infty}\left(\frac{\partial \phi}{\partial t}(x, t)+g\left(x, \zeta_{t}\right) \frac{\partial \phi}{\partial x}(x, t)-\mu\left(x, \zeta_{t}\right) \phi(x, t)\right) d \zeta_{t}(x) d t \\
& +\int_{0}^{T} \phi\left(x_{b}, t\right) \int_{x_{b}}^{\infty} \beta\left(x^{\prime}, \zeta_{t}\right) d \zeta_{t}\left(x^{\prime}\right) d \zeta_{t}(x) d t
\end{aligned}
$$

for all $\phi \in C_{0}^{\infty}\left(\mathbb{R}_{+} \times[0, T]\right)$. Here, $\nu_{0} \in \mathcal{M}_{+}(\Omega)$ is the initial data at time $t=0$.

\footnotetext{
${ }^{1}$ There are two natural notions of convergence on $\mathcal{M}_{+}(\Omega)$ - strong convergence and weak convergence. Strong convergence is unsuitable for our purposes as, for example, the sequence of Dirac measures $\delta_{1 / n}$ does not converge to $\delta_{0}$ as $n \rightarrow \infty$ in the strong topology.
} 
Remark 4. The definition above was inspired by Gwiazda et al. [14]. We differ in that we use smooth test functions, but note that these are dense in the space $C^{1} \cap W^{1, \infty}$ used in [14].

Remark 5. The dependence on the environmental feedback variable $E$ in (1) is represented here by a direct dependence on the solution measure $\zeta_{t}$.

In order to show the convergence of the EBT method, we will recast the definition of a weak solution. Let $0 \leq t_{1}<t_{2} \leq T$ and $v \in \mathcal{M}_{+}(\Omega)$. For a given test function $\phi \in C_{0}^{\infty}\left(\mathbb{R}_{+} \times[0, T]\right)$ and a family of measures $\sigma_{t}$, we define the residual

$$
\begin{aligned}
R_{\phi}\left(\sigma_{t}, \nu, t_{1}, t_{2}\right) & =\int_{x_{b}}^{\infty} \phi\left(x, t_{2}\right) d \sigma_{t_{2}}(x)-\int_{x_{b}}^{\infty} \phi\left(x, t_{1}\right) d \nu(x) \\
& -\int_{t_{1}}^{t_{2}} \int_{x_{b}}^{\infty}\left(\frac{\partial \phi}{\partial t}(x, t)+g\left(x, \zeta_{t}\right) \frac{\partial \phi}{\partial x}(x, t)-\mu\left(x, \zeta_{t}\right) \phi(x, t)\right) d \sigma_{t}(x) d t \\
& +\int_{t_{1}}^{t_{2}} \phi\left(x_{b}, t\right)\left(\int_{x_{b}}^{\infty} \beta\left(x^{\prime}, \zeta_{t}\right) d \sigma_{t}\left(x^{\prime}\right)\right) d t
\end{aligned}
$$

where the measure $\nu$ is interpreted as the initial data at time $t=t_{1}$. Clearly, if $R_{\phi}\left(\sigma_{t}, \nu_{0}, 0, T\right)=0$ for all test functions $\phi$ and the family of measures $\sigma_{t}$ is weakly continuous in time, then $\sigma_{t}$ is a weak solution to (11). We will sometimes write $R_{\phi}\left(\sigma_{t}\right)$ meaning $R_{\phi}\left(\sigma_{t}, \nu_{0}, 0, T\right)$.

\section{Convergence of the Escalator Boxcar Train}

We establish the convergence of the EBT method in five steps: (1) At each fixed time $t$, the sequence of approximating EBT measures contains a subsequence which converges weakly to a positive Radon measure $\zeta_{t}$. (2) We find a subsequence that for all $t$ converges weakly to a mapping $\zeta_{t}$ that is weakly continuous in time. (3) The residuals of the approximating EBT measures $\zeta_{t}^{N}$ converges to the residual of $\zeta_{t}$ for any test function. (4) The residual of the approximating EBT measures $\zeta_{t}^{N}$ converges to zero, and hence the measure $\zeta_{t}$ is a weak solution. All that remains is then to show that the entire sequence of approximating EBT measures converges weakly to $\zeta_{t}$. We do this by (5) assuming the existence of a unique weak solution to the structured population model and showing that a contradiction will otherwise result. In all the following lemmas, we assume that the birth rate, growth rate, and mortality rate are non-negative, bounded, and Lipschitz continuous functions of the individual size $x$. In addition, we need three assumption pertaining to the feedback from the population-level to individual vital rates:

$$
\begin{aligned}
& \sup _{x}|\beta(x, \sigma)-\beta(x, \lambda)| \leq C_{\beta} \rho(\sigma, \lambda), \\
& \sup _{x}|g(x, \sigma)-g(x, \lambda)| \leq C_{g} \rho(\sigma, \lambda), \\
& \sup _{x}|\mu(x, \sigma)-\mu(x, \lambda)| \leq C_{\mu} \rho(\sigma, \lambda) .
\end{aligned}
$$

The three requirements above assert Lipschitz continuity in $\mathcal{M}_{+}(\Omega)$ equipped with the KantorovichRubinstein metric.

Lemma 6 (Step 1). For each $t \in[0, T]$, the sequence $\left\{\zeta_{t}^{N}\right\}$ of approximating EBT measures contains a weakly convergent subsequence. In fact, any subsequence $\left\{\zeta_{t}^{N^{\prime}}\right\}$ of $\left\{\zeta_{t}^{N}\right\}$ contains a weakly convergent subsequence.

Proof. By Prohorov's Theorem [1], it is enough to show that the sequence $\left\{\zeta_{t}^{N}\right\}$ is uniformly bounded in the variation norm and is uniformly tight. As the measures are positive by construction, this amounts to showing that $\zeta_{t}^{N}\left(\left[x_{b}, \infty\right)\right)$ is uniformly bounded in $N$, with $\lim _{M \rightarrow \infty} \sup _{M} \zeta_{t}^{N}((M, \infty))=$ 0 . An biological interpretation of these requirements, which we will build on in the proof, is that 
the abundance and typical size of individuals in the population are bounded from above. Letting $P_{N}(s)=\zeta_{s}^{N}\left(\left[x_{b}, \infty\right)\right)$ it follows that

$$
\begin{aligned}
P_{N}^{\prime}(s) & =\sum_{i=B}^{N} N_{i}^{\prime}(s)=\sum_{i=B}^{N} \beta\left(X_{i}, \zeta_{s}^{N}\right) N_{i}(s)-\sum_{i=B}^{N} \mu\left(X_{i}, \zeta_{s}^{N}\right) N_{i}(s) \leq \\
& \leq \sum_{i=B}^{N} \beta\left(X_{i}, \zeta_{s}^{N}\right) N_{i}(s) \leq \beta_{\text {sup }} \sum_{i=B}^{N} N_{i}(s)=\beta_{\text {sup }} P_{N}(s),
\end{aligned}
$$

where $\beta_{\text {sup }}$ is the supremum of $\beta$, i.e., the maximum individual birth rate. The above inequality holds for all $s \in[0, T]$ except at the finite number of times, where boundary cohorts are internalized. At these points, the function $P_{N}$ is continuous. Thus, $0 \leq P_{N}(t) \leq P_{N}(0) \exp \left(\beta_{\sup } T\right)$. Hence $P_{N}(t)=\zeta_{t}^{N}\left(\left[x_{b}, \infty\right)\right)$ is uniformly bounded on $[0, T]$, since $P_{N}(0)$ is independent of $N$. (Recall that in Sect. 3 we assumed that the initial mass should be independent of $N$ and equal to that of the population measure given as initial condition.)

To prove $\lim _{M \rightarrow \infty} \sup _{N} \zeta_{t}^{N}((M, \infty))=0$, we first show that the statement is true for $t=0$. Let $\varepsilon>0$ be given. Since the initial data $\nu_{0}$ is a positive Radon measure and thus tightly controlled at infinity, we may choose $M_{1}$ large enough such that $\nu_{0}\left(\left(M_{1}, \infty\right)\right)<\varepsilon / 2$. Pick any continuos function $\varphi$ on $\left[x_{b}, \infty\right)$ satisfying $0 \leq \varphi(x) \leq 1$ with $\varphi(x)=1$ for $x>M_{1}+1$ and $\varphi(x)=0$ for $x<M_{1}$. Then

$$
\zeta_{0}^{N}\left(\left[M_{1}+1, \infty\right)\right) \leq \int_{M_{1}}^{\infty} \varphi d \zeta_{0}^{N}<\int_{M_{1}}^{\infty} \varphi d \nu_{0}+\varepsilon / 2<\varepsilon,
$$

if we choose $N>N_{0}$ for some sufficiently large $N_{0}$, since $\zeta_{0}^{N}$ converges weakly to $\nu_{0}$ as $N \rightarrow \infty$. To account for the measures with $N \leq N_{0}$, we choose $M_{2}$ so large that $\zeta_{0}^{N}\left(\left[M_{2}, \infty\right)\right)<\varepsilon$ for $N=$ $1,2, \ldots, N_{0}$. Finally, we choose $M$ as the largest of the two numbers $M_{1}+1$ and $M_{2}$.

To prove the statement for a general time $t \in[0, T]$, we first note that the center of mass and abundance at time $t$ of any internal cohort $i>0$ with $X_{i}(t)$ large enough can be estimated with their respective values at time $t=0$. Specifically, $X_{i}(t) \leq X_{i}(0)+t g_{\text {sup }}$, where $g_{\text {sup }}$ is the supremum of the growth rate $g$, and $N_{i}(t) \leq N_{i}(0)$. Combining these two estimates, we have that $\zeta_{t}^{N}(M, \infty) \leq \zeta_{0}^{N}\left(M-t g_{\text {sup }}, \infty\right)$ and the first assertion of the lemma follows. Finally we note that the above argument holds for any subsequence of $\left\{\zeta_{t}^{N}\right\}$. This concludes the proof.

Lemma 7 (Step 2). The approximating EBT sequence $\left\{\zeta_{t}^{N}\right\}$ contains a subsequence which, for each $t \in[0, T]$, converges weakly to a positive finite measure $\zeta_{t}$. The mapping $\zeta_{t}:[0, T] \rightarrow \mathcal{M}_{+}(\Omega)$ is weakly continuous in time.

Proof. Let $\left\{q_{k}\right\}_{k=1}^{\infty}$ be an enumeration of the rational numbers in $[0, T]$. According to Lemma 6 there exists a convergent subsequence $\left\{\zeta_{q_{1}}^{N_{j}^{1}}\right\}$ of $\left\{\zeta_{q_{1}}^{N}\right\}$. Repeating this argument, there exists a convergent subsequence $\left\{\zeta_{q_{2}}^{N_{j}^{2}}\right\}$ of $\left\{\zeta_{q_{2}}^{N_{j}^{1}}\right\}$. Proceeding by induction, we obtain for each $k$ a sequence $\left\{\zeta_{q_{k}}^{N_{j}^{k}}\right\}$ which converges weakly to $\zeta_{q_{k}}$ and is a subsequence of all preceding sequences. Inspired by Cantor's diagonalization argument we define the sequence $\hat{\zeta}_{t}^{k}:=\zeta_{t}^{N_{k}^{k}}$. It follows that for each rational $t \in[0, T]$, this sequence converges weakly to a measure $\zeta_{t}$.

We will now show that the subsequence also converges to a positive finite Radon measure for all real $t \in[0, T]$. We first show that for each fixed test function $\phi \in C^{\infty}(\mathbb{R})$ and each time $t$, the sequence of real numbers

$$
\int_{x_{b}}^{\infty} \phi d \hat{\zeta}_{t}^{k}
$$

converges as $k \rightarrow \infty$. It then follows from classical results in the theory of distributions, e.g., [17, Theorem 2.1.8 and Theorem 2.1.9], that $\hat{\zeta}_{t}^{k}$ converges weakly to a positive measure $\zeta_{t}$. This will turn out to be the desired measure. 
To prove convergence of the sequence (7), we first note that for fixed $k$, the measure $\hat{\zeta}_{t}^{k}$ is weakly continuous in time since each $N_{i}($.$) and X_{i}($.$) are continuous functions. Let t \in[0, T]$ and $\phi$ be a test function. Given $\varepsilon>0$ we get

$$
\begin{gathered}
\left|\int_{x_{b}}^{\infty} \phi d \hat{\zeta}_{t}^{j}-\int_{x_{b}}^{\infty} \phi d \hat{\zeta}_{t}^{k}\right| \leq \\
\leq\left|\int_{x_{b}}^{\infty} \phi d \hat{\zeta}_{t}^{j}-\int_{x_{b}}^{\infty} \phi d \hat{\zeta}_{q}^{j}\right|+\left|\int_{x_{b}}^{\infty} \phi d \hat{\zeta}_{q}^{j}-\int_{x_{b}}^{\infty} \phi d \hat{\zeta}_{q}^{k}\right|+\left|\int_{x_{b}}^{\infty} \phi d \hat{\zeta}_{q}^{k}-\int_{x_{b}}^{\infty} \phi d \hat{\zeta}_{t}^{k}\right|,
\end{gathered}
$$

for any $j, k$, and $q$. Noting that the birth rate and mortality rate are bounded, we can use the same argument as in the proof of Lemma 6 to show that the first and last term above are bounded by a constant multiple of $|t-q|$. In particular, this constant depends on neither $j$ nor $k$. Choosing $q$ as a rational number sufficiently close to $t$ these two terms will be smaller than $\varepsilon / 2$. Finally, since $q$ is rational, we may choose $j$ and $k$ large enough to make the middle term less than $\varepsilon / 2$. Thus, we have established the Cauchy property for the sequence (7), which hence converges for all test functions $\phi$. This shows that $\hat{\zeta}_{t}^{j}$ converges weakly to a bounded positive Radon measure $\hat{\zeta}_{t}$ for all $t \in[0, T]$.

Using the same idea as above, we see that $\hat{\zeta}_{t}$ is weakly continuous in time. Specifically,

$$
\begin{gathered}
\left|\int_{x_{b}}^{\infty} \phi d \hat{\zeta}_{s}-\int_{x_{b}}^{\infty} \phi d \hat{\zeta}_{t}\right| \leq \\
\leq\left|\int_{x_{b}}^{\infty} \phi d \hat{\zeta}_{s}-\int_{x_{b}}^{\infty} \phi d \hat{\zeta}_{s}^{k}\right|+\left|\int_{x_{b}}^{\infty} \phi d \hat{\zeta}_{s}^{k}-\int_{x_{b}}^{\infty} \phi d \hat{\zeta}_{t}^{k}\right|+\left|\int_{x_{b}}^{\infty} \phi d \hat{\zeta}_{t}^{k}-\int_{x_{b}}^{\infty} \phi d \hat{\zeta}_{t}\right|,
\end{gathered}
$$

where again the middle term is bounded by a constant multiple of $|t-s|$ independent of $k$. Finally, the first and last term can be made arbitrarily small as a consequence of the weak convergence of $\hat{\zeta}_{s}^{k}$ to $\hat{\zeta}_{s}$.

Lemma 8. Assume that the sequence $\zeta_{t}^{k}$ converges weakly to a finite Radon measure $\zeta_{t}$. If $\varphi \in$ $C_{0}^{\infty}\left(\mathbb{R}_{+} \times[0, T]\right)$ then, for every bounded Lipschitz continuous function $f$ satisfying

$$
\sup _{x}|f(x, \sigma)-f(x, \lambda)| \leq C_{f} \rho(\sigma, \lambda),
$$

for all $\sigma, \lambda \in \mathcal{M}_{+}(\Omega)$, we get

$$
\int_{0}^{T} \int_{x_{b}}^{\infty} \varphi(x, t) f\left(x, \zeta_{t}^{k}\right) d \zeta_{t}^{k}(x) d t \rightarrow \int_{0}^{T} \int_{x_{b}}^{\infty} \varphi(x, t) f\left(x, \zeta_{t}\right) d \zeta_{t}(x) d t
$$

as $k$ tends to infinity.

Proof. We have

$$
\begin{aligned}
\int_{x_{b}}^{\infty} \varphi(x, t) f\left(x, \zeta_{t}^{k}\right) d \zeta_{t}^{k}(x) & =\int_{x_{b}}^{\infty} \varphi(x, t) f\left(x, \zeta_{t}\right) d \zeta_{t}^{k}(x)+ \\
& +\int_{x_{b}}^{\infty} \varphi(x, t)\left(f\left(x, \zeta_{t}^{k}\right)-f\left(x, \zeta_{t}\right)\right) d \zeta_{t}^{k}(x) .
\end{aligned}
$$

In the first term on the right hand side, the function $\varphi(x, t) f\left(x, \zeta_{t}\right)$ is bounded and Lipschitz continuous in $x$. Hence, it can be approximated by a sequence $\left\{\varphi_{m}\right\}$ of functions in $C_{0}^{\infty}\left(\mathbb{R}_{+} \times[0, T]\right)$ that converges pointwise and in $W^{1, \infty}$-norm. Such a sequence can, for example, be constructed through convolution. As the first term would vanish if $\left\|\varphi(\cdot, t) f\left(\cdot, \zeta_{t}\right)\right\|_{W^{1, \infty}}=0$, we can assume that this is not the case. We then get

$$
\begin{aligned}
\int_{x_{b}}^{\infty} \varphi(x, t) f\left(x, \zeta_{t}\right) d \zeta_{t}^{k}(x) & =\int_{x_{b}}^{\infty} \lim _{m \rightarrow \infty} \varphi_{m}(x, t) d \zeta_{t}^{k}(x)= \\
& =\lim _{m \rightarrow \infty} \int_{x_{b}}^{\infty} \varphi_{m}(x, t) d \zeta_{t}^{k}(x),
\end{aligned}
$$


where we have used Lebesgue's dominated convergence theorem. Hence,

$$
\begin{gathered}
\left|\int_{x_{b}}^{\infty} \varphi(x, t) f\left(x, \zeta_{t}\right) d \zeta_{t}^{k}(x)-\int_{x_{b}}^{\infty} \varphi(x, t) f\left(x, \zeta_{t}\right) d \zeta_{t}(x)\right|= \\
=\lim _{m \rightarrow \infty}\left|\int_{x_{b}}^{\infty} \varphi_{m}(x, t) d\left(\zeta_{t}^{k}-\zeta_{t}\right)(x)\right|= \\
\leq \lim _{m \rightarrow \infty}\left\|\varphi_{m}(., t)\right\|_{W^{1, \infty}} \rho\left(\zeta_{t}^{k}-\zeta_{t}\right)= \\
=\left\|\varphi(., t) f\left(., \zeta_{t}\right)\right\|_{W^{1, \infty}} \rho\left(\zeta_{t}^{k}-\zeta_{t}\right) \rightarrow 0,
\end{gathered}
$$

as $k$ tends to infinity. Thus the first term converges to

$$
\int_{x_{b}}^{\infty} \varphi(x, t) f\left(x, \zeta_{t}\right) d \zeta_{t}(x)
$$

It remains to show that the second term in (8) vanishes as $k \rightarrow \infty$,

$$
\begin{gathered}
\left|\int_{x_{b}}^{\infty} \varphi(x, t)\left(f\left(x, \zeta_{t}^{k}\right)-f\left(x, \zeta_{t}\right)\right) d \zeta_{t}^{k}(x)\right| \leq \\
\leq \sup _{x}\left|\varphi(x, t)\left(f\left(x, \zeta_{t}^{k}\right)-f\left(x, \zeta_{t}\right)\right)\right| \zeta_{t}^{k}\left(\left[x_{b}, \infty\right)\right) \leq \\
\leq \sup _{x}|\varphi(x, t)| \sup _{x}\left|f\left(x, \zeta_{t}^{k}\right)-f\left(x, \zeta_{t}\right)\right| \zeta_{t}^{k}\left(\left[x_{b}, \infty\right)\right) \leq \\
\leq C_{\varphi} C_{f} \rho\left(\zeta_{t}^{k}, \zeta_{t}\right) \zeta_{t}^{k}\left(\left[x_{b}, \infty\right)\right) .
\end{gathered}
$$

Since $\zeta_{t}^{k}$ converges weakly to $\zeta_{t}$, it follows from Gwiazda et al. [14, Theorem 2.7] that $\zeta_{t}^{k}\left(\left[x_{b}, \infty\right)\right)$ is uniformly bounded and $\rho\left(\zeta_{t}^{k}, \zeta_{t}\right)$ tends to zero as $k$ tends to infinity. Since the above calculation is done pointwise in $t$, the lemma follows from Lebesgue's dominated convergence theorem.

Lemma 9 (Step 3). Assume that the sequence $\zeta_{t}^{k}$ converges weakly to the finite Radon measure $\zeta_{t}$. Then the residual $R_{\phi}\left(\zeta_{t}^{k}\right)$ converges to $R_{\phi}\left(\zeta_{t}\right)$ for all test functions $\phi \in C_{0}^{\infty}\left(\mathbb{R}_{+} \times[0, T]\right)$.

Proof. Consider

$$
\begin{aligned}
R_{\phi}\left(\zeta_{t}^{k}\right)= & \int_{x_{b}}^{\infty} \phi(x, T) d \zeta_{t}^{k}(x)-\int_{x_{b}}^{\infty} \phi(x, 0) d \nu_{0}(x) \\
& -\int_{0}^{T} \int_{x_{b}}^{\infty}\left(\frac{\partial \phi}{\partial t}(x, t)+g\left(x, \zeta_{t}^{k}\right) \frac{\partial \phi}{\partial x}(x, t)-\mu\left(x, \zeta_{t}^{k}\right) \phi(t, x)\right) d \zeta_{t}^{k}(x) d t \\
+ & \int_{0}^{T} \phi\left(x_{b}, t\right)\left(\int_{x_{b}}^{\infty} \beta\left(x^{\prime}, \zeta_{t}^{k}\right) d \zeta_{t}^{k}\left(x^{\prime}\right)\right) d t=I-I I-I I I+I V .
\end{aligned}
$$

The first term converges by definition of weak convergence and the second term is unchanged. The third and fourth term converge by Lemma 8 .

Lemma 10. Let $0 \leq t_{1}<t_{2} \leq T$ and $v \in \mathcal{M}_{+}(\Omega)$. Assuming that no internalization is done in the interval $\left(t_{1}, t_{2}\right)$, then for any test function $\phi$ we have that

$$
\begin{aligned}
R_{\phi}\left(\zeta_{t}^{N}, \nu, t_{1}, t_{2}\right) & =\sum_{i=B}^{N} N_{i}\left(t_{1}\right) \phi\left(X_{i}\left(t_{1}\right), t_{1}\right)-\int_{x_{b}}^{\infty} \phi\left(x, t_{1}\right) d \nu(x)+ \\
& +\int_{t_{1}}^{t_{2}}\left(\phi\left(X_{B}(t), t\right)-\phi\left(x_{b}, t\right)\right) \sum_{i=B}^{N} \beta\left(X_{i}(t), \zeta_{t}^{N}\right) N_{i}(t) d t
\end{aligned}
$$

where the sum is taken over all cohorts, including the boundary cohort. 
Proof. We write the residual (6) as

$$
\begin{aligned}
R_{\phi}\left(\zeta_{t}^{N}, \nu, t_{1}, t_{2}\right) & =\int_{x_{b}}^{\infty} \phi\left(x, t_{2}\right) d \zeta_{t_{2}}^{N}(x)-\int_{x_{b}}^{\infty} \phi\left(x, t_{1}\right) d \nu(x) \\
& -\int_{t_{1}}^{t_{2}} \int_{x_{b}}^{\infty}\left(\phi_{2}(\xi, t)+g\left(x, \zeta_{t}^{N}\right) \phi_{1}(x, t)-\mu\left(x, \zeta_{t}^{N}\right) \phi(x, t)\right) d \zeta_{t}^{N}(x) d t \\
& +\int_{t_{1}}^{t_{2}} \phi\left(x_{b}, t\right)\left(\int_{x_{b}}^{\infty} \beta\left(x^{\prime}, \zeta_{t}^{N}\right) d \zeta_{t}^{N}\left(x^{\prime}\right)\right) d t= \\
& =I\left(\zeta_{t_{2}}^{N}\right)-I I(\nu)-\operatorname{III}\left(\zeta_{t}^{N}\right)-I V\left(\zeta_{t}^{N}\right) .
\end{aligned}
$$

Here we have used the shorthand notation $\phi_{1}(\xi, t)=\partial \phi(\xi, t) / \partial x$ and $\phi_{2}(\xi, t)=\partial \phi(\xi, t) / \partial t$.

Recalling that

$$
\zeta_{t}^{N}=\sum_{i=B}^{N} N_{i}(t) \delta_{X_{i}(t)}
$$

we get

$$
I\left(\zeta_{t_{2}}^{N}\right)=\sum_{i=B}^{N} N_{i}\left(t_{2}\right) \phi\left(X_{i}\left(t_{2}\right), t_{2}\right)
$$

$$
\begin{gathered}
I I I\left(\zeta_{t}^{N}\right)=\sum_{i=B}^{N} \int_{t_{1}}^{t_{2}} N_{i}(t)\left(\phi_{2}\left(X_{i}(t), t\right)+g\left(X_{i}(t), \zeta_{t}^{N}\right) \phi_{1}\left(X_{i}(t), t\right)-\mu\left(x_{i}(t), \zeta_{t}^{N}\right) \phi\left(X_{i}(t), t\right)\right) d t= \\
=I I I_{B}\left(\zeta_{t}^{N}\right)+\sum_{i=B+1}^{N} I I I_{i}\left(\zeta_{t}^{N}\right) .
\end{gathered}
$$

Now, by (3), we have

$$
\begin{aligned}
\operatorname{III}_{i}\left(\zeta_{t}^{N}\right) & =\int_{t_{1}}^{t_{2}} N_{i}(t) \phi_{2}\left(X_{i}(t), t\right)+N_{i}(t) \frac{d X_{i}(t)}{d t} \phi_{1}\left(x_{i}(t), t\right)+\frac{d N_{i}(t)}{d t} \phi\left(X_{i}(t), t\right) d t= \\
& =\int_{t_{1}}^{t_{2}} \frac{d}{d t}\left(N_{i}(t) \phi\left(X_{i}(t), t\right)\right) d t=N_{i}\left(t_{2}\right) \phi\left(X_{i}\left(t_{2}\right), t_{2}\right)-N_{i}\left(t_{1}\right) \phi\left(X_{i}\left(t_{1}\right), t_{1}\right) .
\end{aligned}
$$

Thus

$$
I\left(\zeta_{t_{2}}^{N}\right)-\sum_{i=B+1}^{N} I I I_{i}\left(\zeta_{t}^{N}\right)=N_{B}\left(t_{2}\right) \phi\left(X_{B}\left(t_{2}\right), t_{2}\right)+\sum_{i=B+1}^{N} N_{i}\left(t_{1}\right) \phi\left(X_{i}\left(t_{1}\right), t_{1}\right) .
$$

In the same way, but now also using (44), we get

$$
\begin{aligned}
I I I_{B}\left(\zeta_{t}^{N}\right) & =\int_{t_{1}}^{t_{2}} \frac{d}{d t}\left(N_{B}(t) \phi\left(X_{B}(t), t\right)\right)-\phi\left(X_{B}(t), t\right) \sum_{i=B}^{N} \beta\left(X_{i}(t), \zeta_{t}^{N}\right) N_{i}(t) d t= \\
& =N_{B}\left(t_{2}\right) \phi\left(X_{B}\left(t_{2}\right), t_{2}\right)-N_{B}\left(t_{1}\right) \phi\left(X_{B}\left(t_{1}\right), t_{1}\right)- \\
& -\int_{t_{1}}^{t_{2}} \phi\left(X_{B}(t), t\right) \sum_{i=B}^{N} \beta\left(X_{i}(t), \zeta_{t}^{N}\right) N_{i}(t) d t .
\end{aligned}
$$

Since

$$
I V\left(\zeta_{t}^{N}\right)=\int_{t_{1}}^{t_{2}} \phi\left(x_{b}, t\right) \sum_{i=B}^{N} \beta\left(X_{i}(t), \zeta_{t}^{N}\right) N_{i}(t) d t
$$


we have

$$
\begin{aligned}
-I I I_{B}\left(\zeta_{t}^{N}\right)-I V\left(\zeta_{t}^{N}\right) & =-N_{B}\left(t_{2}\right) \phi\left(X_{B}\left(t_{2}\right), t_{2}\right)+N_{B}\left(t_{1}\right) \phi\left(X_{b}\left(t_{1}\right), t_{1}\right)+ \\
& +\int_{t_{1}}^{t_{2}}\left(\phi\left(X_{B}(t), t\right)-\phi\left(x_{b}, t\right)\right) \sum_{i=B}^{N} \beta\left(X_{i}(t), \zeta_{t}^{N}\right) N_{i}(t) d t .
\end{aligned}
$$

Summing up the calculations above, we get

$$
\begin{gathered}
I\left(\zeta_{t_{2}}^{N}\right)-\sum_{i=B+1}^{N} I I I_{i}\left(\zeta_{t}^{N}\right)-I I I_{B}\left(\zeta_{t}^{N}\right)-I V\left(\zeta_{t}^{N}\right)= \\
=N_{B}\left(t_{2}\right) \phi\left(X_{B}\left(t_{2}\right), t_{2}\right)+\sum_{i=B+1}^{N} N_{i}\left(t_{1}\right) \phi\left(X_{i}\left(t_{1}\right), t_{1}\right)-N_{B}\left(t_{2}\right) \phi\left(X_{B}\left(t_{2}\right), t_{2}\right)+ \\
+N_{B}\left(t_{1}\right) \phi\left(X_{B}\left(t_{1}\right), t_{1}\right)+\int_{t_{1}}^{t_{2}}\left(\phi\left(X_{B}(t), t\right)-\phi\left(x_{b}, t\right)\right) \sum_{i=B}^{N} \beta\left(X_{i}(t), \zeta_{t}^{N}\right) N_{i}(t) d t= \\
=\sum_{i=B}^{N} N_{i}\left(t_{1}\right) \phi\left(X_{i}\left(t_{1}\right), t_{1}\right)+\int_{t_{1}}^{t_{2}}\left(\phi\left(X_{B}(t), t\right)-\phi\left(x_{b}, t\right)\right) \sum_{i=B}^{N} \beta\left(X_{i}(t), \zeta_{t}^{N}\right) N_{i}(t) d t .
\end{gathered}
$$

Finally we get that

$$
\begin{aligned}
R_{\phi}\left(\zeta_{t}^{N}, \nu, t_{1}, t_{2}\right) & =\sum_{i=B}^{N} N_{i}\left(t_{1}\right) \phi\left(X_{i}\left(t_{1}\right), t_{1}\right)-\int_{x_{b}}^{\infty} \phi\left(x, t_{1}\right) d \nu(x)+ \\
& +\int_{t_{1}}^{t_{2}}\left(\phi\left(X_{B}(t), t\right)-\phi\left(x_{b}, t\right)\right) \sum_{i=B}^{N} \beta\left(X_{i}(t), \zeta_{t}^{N}\right) N_{i}(t) d t
\end{aligned}
$$

Remark 11. The residual can be interpreted as the sum of the error arising from the discretization of the initial data and the error arising from the boundary cohort. In the interior of the individual state space, the EBT method gives an exact solution, i.e., there are no errors arising from the transportation of the interior cohorts.

Lemma 12 (Step 4). With $\zeta_{t}^{N}$ defined by the EBT method with internalizations at times $t_{i}=i T / n$, we have that

$$
R_{\phi}\left(\zeta_{t}^{N}, \nu_{0}, 0, T\right) \rightarrow 0,
$$

as $N$ and $n$ tends to infinity. Here $\nu_{0}$ is the initial data at time $t=t_{0}=0$.

Proof. We first write

$$
R_{\phi}\left(\zeta_{t}^{N}, \nu_{0}, 0, T\right)=R_{\phi}\left(\zeta_{t}^{N}, \nu_{0}, 0, t_{1}\right)+\sum_{i=1}^{n-1} R_{\phi}\left(\zeta_{t}^{N}, \zeta_{t_{i}}^{N}, t_{i}, t_{i+1}\right) .
$$

By Lemma 10 we have,

$$
\begin{aligned}
R_{\phi}\left(\zeta_{t}^{N}, \nu_{0}, 0, t_{1}\right) & =\sum_{i=B}^{N} N_{i}(0) \phi\left(x_{i}(0), 0\right)-\int_{x_{b}}^{\infty} \phi(x, 0) d \nu_{0}(x)+ \\
& +\int_{0}^{t_{1}}\left(\phi\left(X_{B}(t), t\right)-\phi\left(x_{b}, t\right)\right) \sum_{i=B}^{N} \beta\left(X_{i}(t), \zeta_{t}^{N}\right) N_{i}(t) d t
\end{aligned}
$$


and

$$
R_{\phi}\left(\zeta_{t}^{N}, \zeta_{t_{i}}^{N}, t_{i}, t_{i+1}\right)=\int_{t_{i}}^{t_{i+1}}\left(\phi\left(X_{B}(t), t\right)-\phi\left(x_{b}, t\right)\right) \sum_{j=B}^{N} \beta\left(x_{j}(t), \zeta_{t}^{N}\right) N_{j}(t) d t
$$

A straightforward estimate now gives

$$
\begin{aligned}
\left|R_{\phi}\left(\zeta_{t}^{N}, \nu_{0}, 0, T\right)\right| & \leq\left|\sum_{i=B}^{N} N_{i}(0) \phi\left(x_{i}(0), 0\right)-\int_{x_{b}}^{\infty} \phi(x, 0) d \nu_{0}(\xi)\right|+ \\
& +\sum_{i=0}^{n-1} \int_{t_{i}}^{t_{i+1}}\left|\phi\left(X_{B}(t), t\right)-\phi\left(x_{b}, t\right)\right| \sum_{j=B}^{N} \beta\left(x_{j}(t), \zeta_{t}^{N}\right) N_{j}(t) d t .
\end{aligned}
$$

The first term tends to zero by assumption as the number of initial cohorts, $N$, tends to infinity. Noting that $x_{b}=X_{B}\left(t_{1}\right)$ and using that the growth rate is bounded, we get

$$
\left|\phi\left(X_{B}(t), t\right)-\phi\left(x_{b}, t\right)\right| \leq C_{\phi}\left|X_{B}(t)-x_{b}\right| \leq C_{\phi g}\left|t-t_{i}\right| .
$$

Hence,

$$
\begin{gathered}
\sum_{i=0}^{n-1} \int_{t_{i}}^{t_{i+1}}\left|\phi\left(X_{B}(t), t\right)-\phi\left(x_{b}, t\right)\right| \sum_{j=B}^{N} \beta\left(x_{j}(t), \zeta_{t}^{N}\right) N_{j}(t) d t \leq \\
\leq \sum_{i=0}^{n-1} C_{\phi g}\left|t_{i+1}-t_{i}\right|^{2} C_{\beta \nu_{0}}
\end{gathered}
$$

for the constant $C_{\beta \nu_{0}}=\beta_{\text {sup }} \nu_{0}\left(\left[x_{b}, \infty\right)\right) \exp \left(\beta_{\text {sup }} T\right)$. Thus, the last sum is bounded by $C(T) / n$ which also tends to zero as the number of internalizations tends to infinity.

Remark 13. Examining the proof above, we see that the residual tends to zero whenever the maximal time between two internalizations of the boundary cohort tends to zero. Hence, we can relax the assumption that the times at which the boundary cohort is internalized are evenly distributed.

Recalling that the initial cohorts are chosen to converge weakly to the initial data, we are now able to prove convergence of the Escalator Boxcar Train:

Theorem 14. Assume that the assumptions on the birth, growth, and mortality rates in the beginning of Sect. 3 hold. If the structured population model given by (1a), (1b), and (1c) has a unique solution $\zeta_{t}$, then the the solutions $\zeta_{t}^{N}$ given by the EBT method converge weakly to $\zeta_{t}$ as the number of initial cohorts tends to infinity and the maximal time between two boundary cohort internalizations tends to zero.

Proof. (Step 5) We assume that the entire sequence $\zeta_{t}^{N}$ does not converge to $\zeta_{t}$. Then, in the weak topology, there exists an open neighborhood $U$ of $\zeta_{t}$, and a subsequence $\zeta_{t}^{N_{k}}$ of $\zeta_{t}^{N}$ such that $\zeta_{t}^{N_{k}} \notin U$ for all $N_{k}$. From Lemma 6, 12, we conclude that $\left\{\zeta_{t}^{N_{k}}\right\}$ contains a convergent sub-sequence with a limit point not equal to $\zeta_{t}$, which is a contradiction since it would imply that the solution to the PSPM is not unique.

The proof of convergence assumed exact solutions to the ordinary differential equations (ODEs) underlying the EBT method. In practical implementations, these need to be solved numerically which introduces small but finite approximation errors. We now extend the convergence proof to account for errors introduced by the underlying ODE solver.

The following lemma is an immediate consequence of Lemma 9 ,

Lemma 15. Assume that $\zeta_{t}^{N, h}=\sum_{i=B}^{N} N_{i}^{h}(t) \delta_{X_{i}^{h}(t)}$. If for each $t$ we have that $N_{i}^{h}(t) \rightarrow N_{i}(t)$ and $X_{i}^{h}(t) \rightarrow X_{i}(t)$ as $h \searrow 0$ then $R_{\phi}\left(\zeta_{t}^{N, h}, \nu_{0}, 0, T\right) \rightarrow R_{\phi}\left(\zeta_{t}^{N}, \nu_{0}, 0, T\right)$ as $h \searrow 0$. 
Combining the lemma above with Theorem 14 we finally have

Theorem 16. Assume that the assumptions on the birth, growth, and mortality rates in the beginning of Sect. 3 hold. If the structured population model given by (1a), (1b), and (1c) has a unique solution $\zeta_{t}$, then the the solutions $\zeta_{t}^{N, h}$, given by the numerical integration of the EBT method, converges weakly to $\zeta_{t}$ if the number of initial cohorts tends to infinity and the maximal time between two boundary cohort internalizations tends to zero, while h tends to zero sufficiently fast.

\section{The original definition of the boundary cohort}

Our study of convergence of the Escalator Boxcar Train in Sect. 3 assumed different dynamics of the boundary cohorts than was used in the original formulation of the method by de Roos [4]. We based our work on the assumption that the boundary cohort differed from the interior cohorts only in the addition of a term for the inflow of newborns. In this section, we consider the convergence of the EBT method under the original definition of the boundary cohort dynamics.

While we simply assumed a dynamical system for the boundary cohort, de Roos formally derived the underlying equations. Consequently, the original dynamics for the boundary cohort reflect the reduction in center of mass that in reality accompanies an inflow of newborns. Moreover, as the center of mass is not defined as a physical quantity for an empty cohort, the equations were derived through series expansion around the size at birth. Thus, rather than tracking the center of mass $X_{B}(t)$ directly, de Roos considered a quantity $\pi_{B}$ which roughly represents the cumulative amount by which the individuals in the boundary cohort exceed their birth size. This quantity is mapped onto the center of mass through the non-linear transformation

$$
X_{B}=\left\{\begin{array}{cc}
\frac{\pi_{B}}{N_{B}}+x_{b}, & \text { if } \pi_{B}>0 \\
x_{b}, & \text { otherwise. }
\end{array}\right.
$$

The specific equations used for defining the boundary cohort were

$$
\begin{aligned}
\frac{d N_{B}}{d t} & =-\mu\left(x_{b}, \zeta^{N}\right) N_{B}-\frac{\partial \mu\left(x_{b}, \zeta^{N}\right)}{\partial x} \pi_{B}+\sum_{i=B}^{N} \beta\left(X_{i}, \zeta^{N}\right) N_{i}, \\
\frac{d \pi_{B}}{d t} & =g\left(x_{b}, \zeta^{N}\right) N_{B}+\frac{\partial g\left(x_{b}, \zeta^{N}\right)}{\partial x} \pi_{B}-\mu\left(x_{b}, \zeta^{N}\right) \pi_{B},
\end{aligned}
$$

with initial conditions $N_{B}=\pi_{B}=0$. We will assume that these are non-negative, as this is a natural requirement which can easily be enforced by an ODE solver if necessary. The appearance of partial derivatives in the expressions above, arising from series expansion around the size at birth, in conjunction with the non-linear transformation mapping $\pi_{B}$ and $N_{B}$ onto $X_{B}$, pose new challenges for proving convergence. As we will show, however, our proof of convergence can be tailored to accompany also the original definition of the boundary cohort.

Note first that the only parts in the proof of convergence in which the equations defining the boundary cohort are used is Lemma 9 and implicitly in Theorem [16. It therefore suffices to give new proofs of these statements. To this end, we require an additional lemma concerning the behavior of the quotient $\pi_{B} / N_{B}$ :

Lemma 17. With $N_{B}$ and $\pi_{B}$ defined by (11) and (12), we get

$$
0 \leq X_{B}-x_{b} \leq C t
$$

for $t \in\left[t_{0}, t_{0}+h\right]$ and some positive constants $C$ and $h$ which only depend on $g, \partial g / \partial x$ and $\partial \mu / \partial x$. 
Proof. From the definitions of $N_{B}$ and $\pi_{B}$ we have

$$
\begin{aligned}
\frac{d}{d t} X_{b} & =\frac{d}{d t} \frac{\pi_{B}}{N_{B}}=\frac{1}{N_{B}} \frac{d \pi_{B}}{d t}-\frac{\pi_{B}}{N_{B}^{2}} \frac{d N_{B}}{d t}= \\
& =g+\frac{\partial g}{\partial x} \frac{\pi_{B}}{N_{B}}-\mu \frac{\pi_{B}}{N_{B}}+\mu \frac{\pi_{B}}{N_{B}}+\frac{\partial \mu}{\partial x} \frac{\pi_{B}^{2}}{N_{B}^{2}}-\frac{\pi_{B}}{N_{B}^{2}} \sum_{i=B}^{N} \beta_{i} N_{i}= \\
& =g+\frac{\partial g}{\partial x} \frac{\pi_{B}}{N_{B}}+\frac{\partial \mu}{\partial x} \frac{\pi_{B}^{2}}{N_{B}^{2}}-\frac{\pi_{B}}{N_{B}^{2}} \sum_{i=B}^{N} \beta_{i} N_{i} \leq \\
& \leq g+\frac{\partial g}{\partial x} \frac{\pi_{B}}{N_{B}}+\frac{\partial \mu}{\partial x}\left(\frac{\pi_{B}}{N_{B}}\right)^{2} .
\end{aligned}
$$

Remembering that $X_{B}=\pi_{B} / N_{B}+x_{b}$, we thus have $X_{B}^{\prime} \leq a+b\left(X_{B}-x_{b}\right)+c\left(X_{B}-x_{b}\right)^{2}$ for some positive constants $a, b$ and $c$. Hence $X_{B}^{\prime} \leq 2 a$ when $X_{B} \leq X_{B}^{*}$ for some positive $X_{B}^{*}$. Since $X_{B}(0)=x_{b}$ it follows that $X_{B}(t) \leq x_{b}+2 a t$ for $t \in\left[0, X_{B}^{*} / 2 a\right]$.

We now use this to show that

Lemma 18. Assume that a new boundary cohort is created at time $t=t_{1}$. For $t_{2}>t_{1}$ sufficiently close to $t_{1}$, we have for all $t \in\left[t_{1}, t_{2}\right]$ that

$$
\left|N_{B}(t)\left(\frac{d X_{B}(t)}{d t}-g\left(X_{B}(t), \zeta_{t}^{N}\right)\right)\right| \leq C_{1}\left(t_{2}-t_{1}\right),
$$

and

$$
\left|\pi_{B}(t) \frac{\partial}{\partial x} \mu\left(X_{B}(t), \zeta_{t}^{N}\right)\right| \leq C_{2}\left(t_{2}-t_{1}\right) .
$$

Proof. Since $N_{B}$ is bounded, $\pi_{B}=N_{B}\left(X_{B}-x_{b}\right)$, it follows from the above proof that $\left|\pi_{B}(t)\right| \leq$ $C\left(t_{2}-t_{1}\right)$ for some positive constant $C$. Hence, since also $\partial \mu\left(X_{B}(t), \zeta_{t}^{N}\right) / \partial x$ is bounded by the assumptions in [4], the statement (14) follows trivially. To show the first part of the assertion, we note that

$$
N_{B}(t)\left(\frac{d X_{B}(t)}{d t}-g\left(X_{B}(t), \zeta_{t}^{N}\right)\right)=\frac{\partial g}{\partial x} \pi_{B}+\frac{\partial \mu}{\partial x} \pi_{B} \frac{\pi_{B}}{N_{B}}-\frac{\pi_{B}}{N_{B}} \sum_{i=B}^{N} \beta_{i} N_{i} .
$$

Since $\pi_{B}$ and $\pi_{B} / N_{B}=X_{B}-x_{b}$ both increases at most linearly from zero, the assertion (13) follows.

The two lemmas above will be used to bound the residual between two internalizations.

Lemma 19. Let $0 \leq t_{1}<t_{2} \leq T$ and $v \in \mathcal{M}_{+}(\Omega)$. For a given test function $\phi \in C_{0}^{\infty}\left(\mathbb{R}_{+} \times[0, T]\right)$ and a family of measures $\sigma_{t}$. Assuming that no internalization is done in the interval $\left(t_{1}, t_{2}\right)$, then

$$
\begin{aligned}
R_{\phi}\left(\zeta_{t}^{N}, \nu, t_{1}, t_{2}\right) & =\sum_{i=B}^{N} N_{i}\left(t_{1}\right) \phi\left(X_{i}\left(t_{1}\right), t_{1}\right)-\int_{x_{b}}^{\infty} \phi\left(x, t_{1}\right) d \nu(x)+ \\
& +\int_{t_{1}}^{t_{2}}\left(\phi\left(X_{B}(t), t\right)-\phi\left(x_{b}, t\right)\right) \sum_{i=B}^{N} \beta\left(X_{i}(t), \zeta_{t}^{N}\right) N_{i}(t) d t+ \\
& +\int_{t_{1}}^{t_{2}} N_{B}(t)\left(\frac{d X_{B}(t)}{d t}-g\left(X_{B}(t), \zeta_{t}^{N}\right)\right) \phi_{1}\left(X_{B}(t), t\right)+ \\
& +\left(\mu_{1}\left(X_{B}(t), \zeta_{t}^{N}\right) \pi_{B}(t)\right) \phi\left(X_{B}(t), t\right) d t
\end{aligned}
$$

where the sums are taken over all cohorts, including the boundary cohort. 
Proof. Examining the proof of Lemma 10 we see that the boundary cohort only appears in the term $I I I_{B}$,

$$
\begin{aligned}
I I I_{B}\left(\zeta_{t}^{N}\right)=\int_{t_{1}}^{t_{2}} N_{B}(t) & \left(\phi_{1}\left(X_{B}(t), t\right)+g\left(X_{B}(t), \zeta_{t}^{N}\right) \phi_{1}\left(X_{B}(t), t\right)-\right. \\
& \left.-\mu\left(X_{B}(t), \zeta_{t}^{N}\right) \phi\left(X_{B}(t), t\right)\right) d t
\end{aligned}
$$

This term is shown to be equivalent with

$$
\int_{t_{1}}^{t_{2}} \frac{d}{d t}\left(N_{B}(t) \phi\left(X_{B}(t), t\right)\right)-\phi\left(X_{B}(t), t\right) \sum_{i=B}^{N} \beta\left(X_{i}(t), \zeta_{t}^{N}\right) N_{i}(t) d t .
$$

Using the original definition for the boundary cohort dynamics, (11) and (12), we derive the required correction term

$$
\begin{gathered}
\int_{t_{1}}^{t_{2}} \frac{d}{d t}\left(N_{B}(t) \phi\left(x_{B}(t), t\right)\right)-\phi\left(X_{B}(t), t\right) \sum_{i=B}^{N} \beta\left(X_{i}(t), \zeta_{t}^{N}\right) N_{i}(t) d t-I I I_{B}\left(\zeta_{t}^{N}\right)= \\
=\int_{t_{1}}^{t_{2}} N_{B}(t)\left(\frac{d X_{B}(t)}{d t}-g\left(x_{B}(t), \zeta_{t}^{N}\right)\right) \phi_{1}\left(X_{B}(t), t\right)+ \\
+\mu_{1}\left(X_{B}(t), \zeta_{t}^{N}\right) \pi_{B}(t) \phi\left(X_{B}(t), t\right) d t .
\end{gathered}
$$

By Lemma 18, we see that the correction term above is bounded by $C\left(t_{2}-t_{1}\right)^{2}$. Analogous to Lemma 12, we then have

Lemma 20. With $\zeta_{t}^{N}$ defined by the EBT method with internalizations at times $t_{i}=i T / n$, we have that

$$
R_{\phi}\left(\zeta_{t}^{N}, \nu_{0}, 0, T\right) \rightarrow 0
$$

as $N$ and $n$ tends to infinity. Here $\nu_{0}$ is the initial data at time $t=t_{0}=0$.

The original definition of the boundary cohorts might prove more challenging from a numerical perspective. However, if we can determine numerically solutions $\zeta_{t}^{N, h}$ to the equations of the EBT method such that the center of mass, $X_{B}^{h}$, now determined by the non linear transformation (10) converges to its true value, $X_{B}$, as the step length $h \searrow 0$, the residual still tends to zero according to Lemma 15. Hence, the numerical convergence follows as before.

\section{Discussion}

Enhanced biological realism and predictive ability of theoretical investigations are gaining importance as anthropogenic impacts are fundamentally altering the native environment of many organisms. Physiologically structured population models (PSPMs) are increasingly used to model and analyze biological systems. As these models account for the physiological development of individuals, they are better able to predict system dynamics. In contrast to simple unstructured population models such as the classical Lotka-Volterra equations, PSPMs often defy analytical investigations due to the non-local dependencies. There is thus a mounting need for numerical methods that can effectively uncover the underlying dynamics. The Escalator Boxcar Train (EBT) has been specifically designed for PSPMs and has three major advantages: it prevents numerical diffusion, it is relatively easy to implement, and the underlying equations allow for a natural biological interpretation. The method was developed more than two decades ago and has been used to study PSPMs ever since, but the 
fact that convergence has never been formally proved might well have hampered its wider acceptance beyond the domains of theoretical biology.

In this paper we have given the first rigorous proof of convergence for the EBT method. Our proof is given in a modern setting of measure-valued solutions (see e.g., [14]). This contrasts with previous efforts by de Roos and Metz [6] that were carried out in a classical setting and thus required additional smoothness assumptions. While their efforts fell short of proving the full convergence of the EBT method, the authors succeeded in showing that the method consistently approximates the true solution, i.e., that the local approximation error as measured through an arbitrary (but smooth) functional of the solution is bounded and vanishes in the limit of infinitely fine discretization of the individual state space.

There are many possible extensions of the work presented here. A straightforward extension is to write down the corresponding proof for a higher-dimensional state space but with a single birth state. We believe that with more tedious calculations, one could prove the convergence also for the case of stochastic birth state. A more challenging extension is to consider stochasticity in individual development. On the population-level, this roughly amounts to diffusion and it is difficult to see how the EBT method should best be adapted to deal with this situation. Here, some inspiration might come from moving-mesh discontinuous Galerkin methods which, at least at first glance, appear to have similarities with the EBT method. A further extension is to consider different formulations of the boundary cohort. We initially proved convergence when the boundary cohort differed only by the addition of a fecundity term. While this works mathematically, it is natural to account for the fact that newborn individuals reduces the average size of individuals in the boundary cohort. The original formulation of the EBT method does account for this through a different definition of the boundary cohort, and as a second step we analyzed and proved convergence for this case. We believe that our proof can be extended to show convergence also for other formulations of the boundary cohort, as long as the flux of individuals is preserved. Analyzing convergence rates for different definitions of boundary cohorts would be an interesting extension of the work presented here. In particular, we believe that the series expansion around the size at birth underlying the original derivation of the boundary cohorts is not required, and that a direct evaluation at the center of mass might lead to even faster convergence. This could well be part of a more broadly encompassing study that explores convergence rates under different smoothness assumptions. A final important extension would be to consider vital rates that depend on the entire history of the population state up to the current time, rather than merely the current population state, as this would encompass cases with dynamic environmental feedback variables.

Given the long tradition of partial differential equations (PDEs) in the physical sciences, it is not surprising that PSPMs were initially studied using this formalism. Efforts in the last decades have revealed, however, that the PDE formalism is not well-suited for considering questions of existence, uniqueness, and stability. For this reason, the cumulative formulation of structured population models [12, 9] was developed. It had the drawback, however, that a principle of linearized stability and the Hopf bifurcation theorem proved hard to establish [16. Currently, it appears that renewal equations are well-suited for studying PSPMs [10, 8, 11, 16]. The work presented here has been developed from the PDE setting. We believe, however, that renewal equations are a promising framework for developing and analyzing numerical methods for PSPMs. A first step would be to recast the EBT method in this setting, after which the extensions outlined above could be considered. With interest in PSPMs now mounting, a historical opportunity exists for bridging biological theory and computational mathematics through the development of modern numerical methods for the 21st century.

Acknowledgement 21. A.B. and D.S. gratefully acknowledge support from the Kempe Foundations. We thank Odo Diekmann, Mats Larson, and Hans Metz for valuable comments and suggestions. 


\section{References}

[1] V. I. Bogachev. Measure theory. Vol. I, II. Springer-Verlag, Berlin, 2007.

[2] C. J. Briggs, R. M. Nisbet, W. W. Murdoch, T. R. Collier, and J.A.J. Metz. Dynamical effects of Host-Feeding in parasitoids. Journal of Animal Ecology, 64(3):403-416, 1995.

[3] J. A. Carrillo, R. M. Colombo, P. Gwiazda, P., and A. Ulikowska. Structured populations, cell growth and measure valued balance laws. J. Differential Eqautions 252(4):3245-3277, 2012.

[4] A. M. de Roos. Numerical methods for structured population models: the escalator boxcar train. Numer. Methods Partial Differential Equations, 4(3):173-195, 1988.

[5] A. M. de Roos. A gentle introduction to models of physiologically structured populations. In S. Tuljapurkar and H. Caswell, editors, Structured-population models in marine, terrestrial, and freshwater systems, pages 119-204. Chapman \& Hall, New York, 1997.

[6] A. M. de Roos and J. A. J. Metz. Towards a numerical analysis of the escalator boxcar train. In Differential equations with applications in biology, physics, and engineering (Leibnitz, 1989), volume 133 of Lecture Notes in Pure and Appl. Math., pages 91-113. Dekker, New York, 1991.

[7] O. Diekmann and Ph. Getto. Boundedness, global existence and continuous dependence for nonlinear dynamical systems describing physiologically structured populations. J. Differential Equations, 215(2):268-319, 2005.

[8] O. Diekmann and M. Gyllenberg. The second Half-With a quarter of a century delay. Mathematical Modelling of Natural Phenomena, 3(7):36-48, October 2008.

[9] O. Diekmann, M. Gyllenberg, H. Huang, M. Kirkilionis, J. A. J. Metz, and H. R. Thieme. On the formulation and analysis of general deterministic structured population models. II. Nonlinear theory. J. Math. Biol., 43(2):157-189, 2001.

[10] O. Diekmann, Ph. Getto, and M. Gyllenberg. Stability and bifurcation analysis of volterra functional equations in the light of suns and stars. SIAM Journal on Mathematical Analysis, 39(4):1023, 2008.

[11] O. Diekmann, M. Gyllenberg, J. A. J. Metz, S. Nakaoka, and A. M. Roos. Daphnia revisited: local stability and bifurcation theory for physiologically structured population models explained by way of an example. J. Math. Biol., 61(2):277-318, 2009.

[12] O. Diekmann, M. Gyllenberg, J. A. J. Metz, and H. R. Thieme. On the formulation and analysis of general deterministic structured population models. I. Linear theory. J. Math. Biol., 36(4):349-388, 1998.

[13] R. Goetz, N. Hritonenko, A. Xabadia, and Y. Yatsenko. Using the escalator boxcar train to determine the optimal management of a Size-Distributed forest when carbon sequestration is taken into account. In Ivan Lirkov, Svetozar Margenov, and Jerzy Wasniewski, editors, Large-Scale Scientific Computing, volume 4818 of Lecture Notes in Computer Science, 334-341. Springer Berlin / Heidelberg, 2008.

[14] P. Gwiazda, T. Lorenz, and A. Marciniak-Czochra. A nonlinear structured population model: Lipschitz continuity of measure-valued solutions with respect to model ingredients. J. Differential Equations, 248(11):2703-2735, 2010.

[15] P. Gwiazda and A. Marciniak-Czochra. Structured population equations in metric spaces. J. Hyperbolic Differ. Equ. 7(4):733-773, 2010. 
[16] M. Gyllenberg. Mathematical aspects of physiologically structured populations: the contributions of j. a. j. metz. J. Biological Dynamics, 1(1):3-44, 2007.

[17] L. Hörmander. The analysis of linear partial differential operators. I. Springer Study Edition. Springer-Verlag, Berlin, second edition, 1990.

[18] J. A. J. Metz and O. Diekmann. Formulating models for structured populations, volume 68 of Lecture Notes in Biomath. Springer, Berlin, 1986.

[19] L. Persson, K. Leonardsson, A. M. de Roos, M. Gyllenberg, and B. Christensen. Ontogenetic scaling of foraging rates and the dynamics of a Size-Structured Consumer-Resource model. Theor. Popul. Biol., 54(3):270-293, 1998.

[20] A. Xabadia and R. U. Goetz. The optimal selective logging regime and the faustmann formula. Journal of Forest Economics, 16(1):63-82, 2010. 